\title{
THE SURFACE ADHESION PARAMETER: A MEASURE FOR WAFER BONDABILITY
}

\author{
C. Gui, M. Elwenspoek, N. Tas, and J.G.E. Gardeniers \\ MESA Research Institute, University of Twente, The Netherlands \\ E-mail: c.gui@el.utwente.nl
}

\begin{abstract}
A theory is presented which describes the initial direct wafer bonding process. The effect of surface microroughness on the bondability is studied on the basis of the theory of contact and adhesion of elastic solids. An effective bonding energy, the maximum of which is the specific surface energy of adhesion, is proposed to describe the real binding energy of the bonding interface including the influence of the wafer surface microroughness. Both the effective bonding energy and the real area of contact between rough surfaces depend on a dimensionless surface adhesion parameter, $\theta$. Using the adhesion parameter as a measure, three kinds of wafer contact interfaces can be identified with respect to their bondability; viz. the nonbonding regime $(\theta>\approx 12)$, the bonding regime $(\theta<\approx 1)$, and the adherence regime $(1<\theta<12)$. Experimental data are in agreement with this theory.
\end{abstract}

\section{INTRODUCTION}

The mechanism of direct wafer bonding at room temperature has been attributed to the short range intermolecular and inter-atomic attraction forces, such as Van der Waals forces [1]. Consequently, the wafer surface smoothness becomes one of the most critical parameters in this process. High surface roughness will result in small real area of contact, and therefore yield voids in the bonding interface. When the surface roughness exceeds a critical value, the wafers will not bond at all.

The first theory on the problem of closing gaps between contacted wafers was proposed by Stengl et al. [2]. This gap-closing theory was then further developed by Tong and Gösele et al $[1,3,4]$. The elastomechanics theory was used to study the energy balance between the released energy during bonding and the energy increase due to the elastic distortion of the wafer. A detailed analysis on the three dimensional elastic field in the misfit between contacted wafers has been presented by $\mathrm{Yu}$ and $\mathrm{Hu}$ very recently [5], resulting in the same results as the gap-closing theory. The gap-closing theory gives a criterion for room temperature wafer bonding. However, the extend of bonding has not been explained; and a few questions remain unclear. For example, what is the real area of contact in the wafer bonding interface after contact at room temperature? The wafer surface always possesses a random distribution of surface topography. If this is taken into account, what will be different in the theory on surface bondability? Therefore we are motivated to develop a continuous model of the influence of surface roughness on wafer bonding, that will be based on contact mechanics theory and a statistical surface roughness model.

\section{DIMENSIONAL ANALYSIS}

As depicted in Fig. 1, there are basically three factors that are relevant to the wafer bonding process at room temperature and at standard atmospheric pressure: the material deformability, $E\left[\mathrm{~N} \cdot \mathrm{m}^{-2}\right]$; the specific surface energy of adhesion, $w\left[\mathrm{~N} \cdot \mathrm{m}^{-1}\right]$; and the surface roughness of both wafers. The wafer surface roughness can be characterised by two variables: length parameter $\lambda[\mathrm{m}]$, and height parameter $h[\mathrm{~m}]$. Example of the length parameter is the dominant wavelength of the wafer surface topography, and that of the height parameter is the standard height deviation of the surface scan.

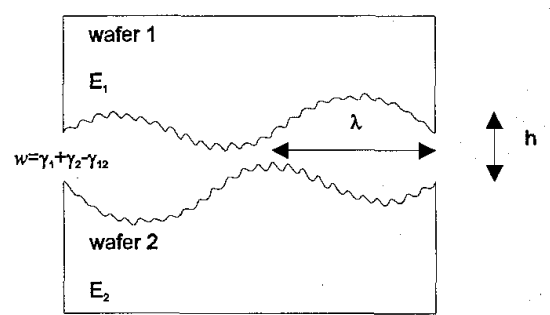

Fig. 1 Cross section of two wafers in contact for direct bonding.

It is clear that bonding will be easier if the surface tension is large and the material is readily deformable. So the criterion must have something to do with the ratio $w / E$, which has the dimension of a length. On the other hand, if the surfaces are smooth ( $h$ small) and the roughness has a large wavelength it is also easy to deform them. The simplest combination of $h$ and $\lambda$ that gives a sensible dimensionless result is $\lambda / h^{2}$. Therefore, we have

$$
\text { Criterion for bondability } \propto \frac{w}{E} \bullet \frac{\lambda}{h^{2}}
$$


If the mean curvature of the asperities, $\beta$, is used to characterise the rough surface, substitution of the relation $\beta \propto \lambda^{2} / h[6]$ into Eq. 1 leads to:

$$
\text { Criterion for bondability } \propto \frac{w}{E} \bullet \sqrt{\frac{\beta}{h^{3}}}
$$

Later we will show that this is exactly the parameter for wafer bondability as derived from the contact mechanics theory.

\section{THEORY}

All wafer surfaces are rough, at least on a microscopic scale. The scan profile of a wafer surface is characterised by many asperities. In many situations, the asperities have spherical caps. During wafer bonding, these asperities on one wafer will first make contact with the surface of the other wafer. Therefore, we shall first study the contact and adhesion of single elastic spheres.

The DMT theory on the contact and adhesion of single elastic spheres

Let us consider the contact between an elastic sphere with high elasticity, such as silicon, and a rigid flat plane under a normal load $P$. The elastic sphere has a radius of $R$. The surface adherence forces act around the periphery of the contact area, and has a value of $2 \pi w R$, as calculated by Derjaguin et al. [7] and Maugis [8]. The distribution of the stresses within the contact regime is assumed to be Hertzian. Thus the radius of contact, $a$, and the elastic displacement $\delta$ at the tip of the sphere are given by $[7,8]$ :

$$
\begin{aligned}
& \frac{a^{3} K}{R}=P-2 \pi R w, \\
& \delta=\frac{a^{2}}{R}
\end{aligned}
$$

where, $K=\frac{4}{3} E^{*}=\frac{4}{3} \frac{E}{1-v^{2}}$, with $v$ the Poisson's ratio and $E$ the Young's modulus of the sphere.

The DMT theory is a modified Hertzian contact theory taking the surface attraction forces into account. This theory is valid for the contact and adhesion between solids with high elasticity $[9,10]$, which is generally the case for the contact and adhesion between silicon wafers. It is clear from Eq. (3), that when the contact radius is zero (point contact), the pull-off force of the contact is $2 \pi w R$. If the applied load is zero, there is a finite radius of contact $a_{0}\left(=\left(2 \pi w R^{2} / K\right)^{1 / 3}\right)$. These are the effects of the adherence forces. It is worthwhile mentioning that the relations between the applied load, the deformation of the tip and the contact radius are unique according to this theory. Therefore, the processes that the elastic sphere is approaching to or is separating from the rigid plane are reversible. Hence, both contact and separation processes will follow the same law as given in Eqs. (3) and (4) [8].

\section{The contact and adhesion between wafers}

We study the contact and adhesion between a wafer with a rough surface and a perfectly smooth rigid wafer (Fig. 2). The surface roughness and the elastic properties of both wafers in a real situation can always be combined on one wafer surface. Here, we consider the effect of the surface microroughness. The rough wafer surface is assumed to be nominally flat. It is assumed that, on the rough wafer surface, there are $N$ asperities within a nominal area of $A_{0}$ (density $\eta_{s}=$ $N / A_{0}$ ). The asperities have spherical caps of the same radius $R$ and a Gaussian height distribution of standard deviation $\sigma$.

$$
\varphi(z)=\frac{1}{\sigma \sqrt{2 \pi}} \exp \left(-\frac{z^{2}}{2 \sigma^{2}}\right)
$$

where $\varphi(z) d z$ is the probability that an asperity has a height between $z$ and $z+d z$ above the plane defined by the mean asperity height.

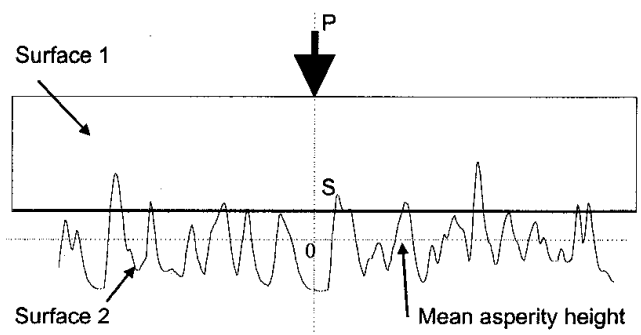

Fig. 2 A rough surface having asperities of Gaussian height distribution in contact with a rigid flat plane. The separation is $s$ when the load is $P$.

As shown by Archard, the quantities $R, \sigma, \eta_{s}$ are not independent but related [6], $R \sigma \eta_{s}=$ Constant . The value of this constant is between 0.05 and 0.1 .

Let us define the situation when the two wafers have been brought into contact under a load $P$; the separation between the rigid flat wafer and the plane of mean asperity height of the wafer with rough surface being $s$. The number of asperities in contact, $n$, can be calculated by:

$$
n=N \int_{s}^{\infty} \varphi(z) d z .
$$

In the case of the $i_{\text {th }}$ asperity, which is in contact with the rigid flat plane, the area of contact, $A_{i}$, and the compressive force, $P_{i}$ can be derived from Eqs. (3) and (4):

$$
\begin{aligned}
& A_{i}=\pi a_{i}^{2}=R \delta_{i}=R\left(z_{i}-s\right) \\
& P_{i}=K R^{1 / 2} \delta_{i}^{3 / 2}-2 \pi w R \\
& =K R^{1 / 2}\left(z_{i}-s\right)^{3 / 2}-2 \pi w R
\end{aligned}
$$


where $z_{i}$ is the height of the $i_{t h}$ asperity. Obviously the total real area of contact will be:

$$
A=\frac{N R}{\sqrt{2 \pi} \sigma} \int_{s}^{\infty}(z-s) \exp \left(-\frac{z^{2}}{2 \sigma^{2}}\right) d z
$$

and the total load is:

$$
P=\frac{N K R^{1 / 2}}{\sqrt{2 \pi} \sigma} \int_{s}^{\infty}(z-s)^{3 / 2} \exp \left(-\frac{z^{2}}{2 \sigma^{2}}\right) d z-\frac{2 \pi w R N}{\sqrt{2 \pi} \sigma} \int_{s}^{\infty} \exp \left(-\frac{z^{2}}{2 \sigma^{2}}\right) d z
$$

Let us introduce a dimensionless parameter, $\theta$ :

$$
\theta=\frac{E^{*}}{w} \sqrt{\frac{\sigma^{3}}{R}} .
$$

This parameter was first given by Fuller and Tabor [11] and is known as the adhesion parameter. Using the adhesion parameter, and replacing $s$ with $s^{*}(=s / \sigma), z$ with $x(=z / \sigma)$, we can rewrite Eqs. (9) and (10) as:

$$
\begin{aligned}
& A^{*}=\frac{A}{A_{0}}=\pi R \sigma \eta_{s} \int_{s^{*}}^{\infty}\left(x-s^{*}\right) \exp \left(-\frac{x^{2}}{2}\right) d x, \\
P^{*}= & \frac{P}{A_{0} w / \sigma} \\
= & R \sigma \eta_{s}\left(\frac{4 \theta}{3 \sqrt{2 \pi}} \int_{s^{*}}^{\infty}\left(x-s^{*}\right)^{3 / 2} \exp \left(-\frac{x^{2}}{2}\right) d x-\sqrt{2 \pi} \int_{s^{*}}^{\infty} \exp \left(-\frac{x^{2}}{2}\right) d x\right)
\end{aligned}
$$

where $A^{*}$ is the normalised real area of contact, and $P^{*}$ is the normalised contact force. Similar equations (Eqs. (12) and (13)) have also been derived by Maguis [10].

Eq. (13) gives the relation between the normalised contact force and the normalised separation of the two contacted wafer surfaces. After numerical integration, this relation is graphically shown in Fig. 3.

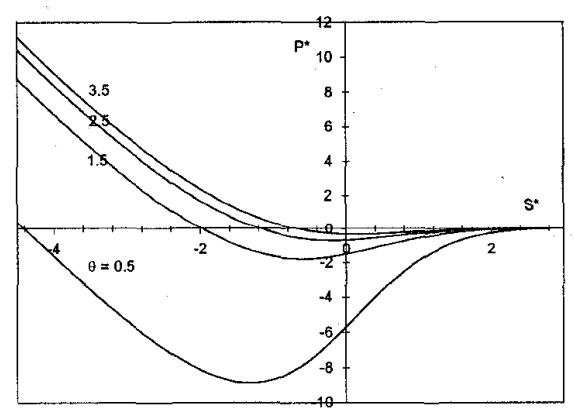

Fig. 3. The normalised force $P^{*}$ as a function of the normalised separation $s^{*}$ for various parameters $\theta$ in the case of elastic contact between a rough wafer surface and a flat wafer surface (DMT-Maugis contact theory).

It is observed from Fig. 3 that there are equilibrium separations $s_{b}{ }_{b}$, at which the surface separation forces induced by the elastic deformation and the surface attraction forces due to the surface adhesion are balanced. If the separations are larger than $s_{b}^{*}$, pull forces or separation forces are necessary to maintain the separation. Should the separations be smaller than $s_{b}{ }_{b}$, push forces are needed to maintain the closer distances between two surfaces. The equilibrium separation $s^{*}{ }_{b}$ is a function of the surface adhesion parameter $\theta$.

\section{The real area of contact during wafer bonding}

If the gravitation force is disregarded, the total load applied on the wafers at the equilibrium condition of wafer bonding will be zero, i.e. $P=0$. Thus the separation between two wafers, $s_{b}{ }^{*}$, at the bonding situation can be solved from Eq. (13). The separation at the bonding equilibrium will be a function of the adhesion parameter. Let us write:

$$
s_{b}^{*}=G\left(\theta^{*}\right) \text {. }
$$

Substituting $s_{b}{ }^{*}$ into Eq. (12), we obtain the real area of contact in the bonding interface between a rough wafer surface and a flat wafer surface:

$$
A_{b}^{*}=\frac{A_{b}}{A_{0}}=\pi R \sigma \eta_{s} \int_{s_{b}^{*}}^{\infty}\left(x-s_{b}^{*}\right) \exp \left(-\frac{x^{2}}{2}\right) d x
$$

where $A_{b}$ is the real area of contact in the bonding interface, and $A_{b}{ }^{*}$ is the normalised real area of contact in the bonding interface. A graphical representation of the relation between the real area of contact and the adhesion parameter is shown in Fig. 4.

\section{The effective bonding energy}

After bonding of two wafers, energy is needed to separate them. The mechanical work needed for separating two bonded surfaces from an equilibrium distance of $s_{b}$ to a distance of infinity, $U_{\text {seperation }}$, is given by:

$$
U_{\text {separation }}=\int_{s_{b}}^{\infty} P d s
$$

We define the mechanical work needed for separating per unit nominal area of contact interface as the specific effective bonding energy, $w_{b}$. This specific effective bonding energy is given by:

$$
w_{b}=\frac{U_{\text {separation }}}{A_{0}}=\frac{\int_{s_{b}}^{\infty} P d s}{A_{0}} .
$$

Substituting Eqs. (10) and (13) into Eq. (17), we have:

$$
\begin{aligned}
& w_{b}^{*}=\frac{w_{b}}{w} \\
& =R \sigma \eta_{s} \int_{s_{b}^{*}}^{\infty}\left(\frac{4 \theta}{3 \sqrt{2 \pi}} \int_{s^{*}}^{\infty}\left(x-s^{*}\right)^{3 / 2} \exp \left(-\frac{x^{2}}{2}\right) d x-\sqrt{2 \pi} \int_{s^{*}}^{\infty} \exp \left(-\frac{x^{2}}{2}\right) d x\right) d s^{*}
\end{aligned}
$$


where $w_{b}{ }^{*}$ is the normalised specific effective bonding energy.

The normalised specific effective bonding energy, as a function of the adhesion parameter, is graphically shown in Fig. 4.

The maximum limit of the real area of contact is the apparent area of the surface. Similarly, the maximum limit of the specific effective bonding energy is the specific surface energy of adhesion. This specific surface energy of adhesion depends on the surface chemistry [12], and can be calculated by taking into account each possible molecular bond on a flat surface. For example, in the case of direct bonding of hydrophilic silicon wafers, the specific surface energy of adhesion will be about $0.1 \mathrm{~J} / \mathrm{m}^{2}$ if the maximum density of the hydrogen bonds is taken into account [13]. In the wafer bonding practice, the wafer surfaces can be modified such that higher specific surface energies of adhesion are possible. However, the specific effective bonding energy can never be higher than the specific surface energy of adhesion, unless further treatments are carried out to strengthen the effective bonding energy.

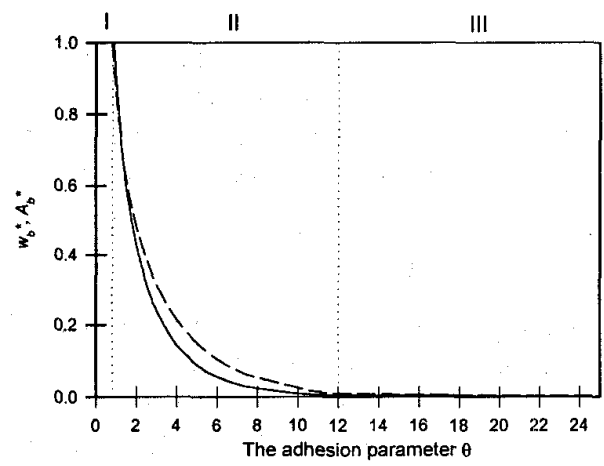

Fig. 4. The normalised specific effective bonding energy (solid line) and the normalised real area of contact (dashed line) as functions of the surface adhesion parameter. The contacted surfaces are a rough surface with Gaussian height distribution and a flat plane. Using the adhesion parameter as a measure, three regimes can be identified: $I$, the bonding regime for $\theta<\approx 1$; II, the adherence regime for $1<\theta<12$; and III, the non-bonding regime for $\theta>\approx 12$.

\section{The surface criterion for direct wafer bonding}

Up to now, there is no clear definition for the bondability of surfaces in terms of direct wafer bonding. However, it seems that the bonding energy, the real area of contact and/or the bond wave propagation speed are the most relevant parameters. The bonding energy/strength and the contact area are useful, for instance, in characterising the hermetic sealing performance of the bonding required in many applications. Also, the voids induced by the surface roughness are directly related to the real area of contact. For a good direct wafer bonding, the bonding energy and the real area of contact must be as large as possible. Ideally the specific effective bonding energy reaches the theoretical specific energy of adhesion, and the contact is $100 \%$. The bonding energy and the real area of contact are closely related to each other, which can be clearly seen from Fig. 4. A higher real area of contact is accompanied with a higher effective specific bonding energy. In practice, it is usually observed that a spontaneous contact between two wafers will result in a good bonding, viz. large contact area and high bond energy.

Here, we shall use the real area of contact and the specific effective bonding energy as characteristics for the bondability in direct wafer bonding. In general, three regimes can be identified from Fig. 4 . When the adhesion parameter is larger than, say 12 , both the effective bonding energy and the real area of contact are practically zero. We call this the non-bonding regime. When the adhesion parameter is smaller than about 1 , full area of contact and full bonding energy is achieved. We call this the bonding regime. Between the bonding regime and the non-bonding regime, there is a transition regime, called the adherence regime. In the adherence regime, the real area of contact and the effective bonding energy vary with the adhesion parameter. Recalling the definition of the surface adhesion parameter, it can be concluded that surfaces with smaller roughness, higher specific energy of adhesion, and/or lower material elasticity will lead to a higher percentage of real area of contact and a higher bond energy.

It is worthwhile mentioning that the regime of nonbonding does not necessary mean that it is a regime free of stiction. For the contact between a micromechanical structure, e.g. a cantilever beam, and the substrate surface, stiction may occur even when $\theta \geq 12$, because the adherence force may be large enough to overcome the restoring elastic force.

\section{EXPERIMENTAL AND RESULTS}

In order to examine the theory, experiments on the direct bonding of wafers having different surface roughness have been carried out. Five pairs of primary grade 3" P-doped $<100>$ silicon wafers were used. The primary grade polished surfaces of six pieces of wafers were kept as original, while the surfaces of the other four pieces of wafers were modified by means of chemical mechanical polishing (CMP) and chemical etching (Table 1). CMP was carried out with an PRESI E460 polishing machine which has a single polisher. Using various polishing pads and slurries, the surface roughnesses of different levels can be obtained [14]. Wet chemical etching processes, such as HF etching and $\mathrm{KOH}$ etching, were also used in the modification of wafer surfaces. After CMP and chemical etching, wafers were cleaned in an $\mathrm{H}_{2} \mathrm{SO}_{4} / \mathrm{H}_{2} \mathrm{O}_{2} / \mathrm{H}_{2} \mathrm{O} \cdot 1: 1: 5$ solution at $80^{\circ} \mathrm{C}$ for 20 minutes, to remove 
contamination introduced in the polishing and chemical etching processes.

Then, the wafers were cleaned with fuming nitric acid $(100 \%)$ and hot nitric acid $\left(70 \%\right.$ at $\left.90^{\circ} \mathrm{C}\right)$. After each cleaning step, a Quick Dump Rinse (QDR) with deionised (DI) water was carried out. Prior to bonding, the wafers were treated in a so-called Piranha solution at $100^{\circ} \mathrm{C}$ for 30 minutes. The Piranha solution consists of three parts $\mathrm{H}_{2} \mathrm{SO}_{4}$ and one part $\mathrm{H}_{2} \mathrm{O}_{2}$. The wafers were kept wet in the QDR, rinsing several times, and spindried only just before bonding. After these treatments, the wafers will possess hydrophilic surfaces, which will provide specific adhesion energy of about $0.1 \mathrm{~J} / \mathrm{m}^{2}$, assuming that the surfaces are very smooth $[1,13]$.

Table 1 Processes for modification of the wafer surfaces and the results of surface roughness characterisations

\begin{tabular}{|c|c|c|c|c|c|}
\hline No. & $\begin{array}{l}\text { Surface } \\
\text { modification }\end{array}$ & $\begin{array}{l}\sigma \\
{[\mathrm{nm}]}\end{array}$ & $\begin{array}{l}\mathbf{R} \\
{[\mu \mathrm{m}]}\end{array}$ & $\begin{array}{l}\eta_{\mathrm{s}} \\
{\left[\mu \mathrm{m}^{-2}\right]}\end{array}$ & $\sigma \mathrm{R} \eta_{\mathrm{s}}$ \\
\hline 6 & $\begin{array}{l}\text { CMP, Pad: IC } \\
\text { 1000/ SUBA IV } \\
\text { Slurry: } \\
\text { Nalco2350/ DI } \\
\mathrm{H}_{2} \mathrm{O} 1: 30\end{array}$ & 1.2 & $\begin{array}{l}10.9 \\
\pm 4.6\end{array}$ & 6.25 & 0.08 \\
\hline 7 & $\begin{array}{l}\text { CMP, Pad: IC } \\
1000 \text { / SUBA IV } \\
\text { Slurry: } \\
\text { Semisperse25/ DI } \\
\mathrm{H}_{2} \mathrm{O} 1: 2\end{array}$ & 1.1 & $\begin{array}{l}13.3 \\
\pm 5.1\end{array}$ & 4.41 & 0.07 \\
\hline 8 & $\begin{array}{l}\text { HF (1 \%) etching, } \\
60 \mathrm{sec} \text {. } \\
\mathrm{KOH}(33 \%) \\
\text { etching } 30 \mathrm{sec} \text {. }\end{array}$ & 1.0 & $\begin{array}{l}1.8 \pm \\
0.5\end{array}$ & 38.4 & 0.07 \\
\hline 9 & $\begin{array}{l}\text { HF (1 \%) etching, } \\
60 \mathrm{sec} . \\
\mathrm{KOH} \quad(33 \%) \\
\text { etching } 10 \text { sec. }\end{array}$ & 0.9 & $\begin{array}{l}2.0 \pm \\
0.8\end{array}$ & 33.6 & 0.06 \\
\hline 10 & No & $<0.1$ & $\begin{array}{l}76.2 \\
\pm 43\end{array}$ & 21.2 & 0.07 \\
\hline
\end{tabular}

Note: $1 . \sigma, R, \eta_{s}$ were measured and calculated from one cross section from the $10 \times 10 \mathrm{\mu m}^{2}$ AFM scan.

2. The standard height deviation, the mean cap radius and the density, and all of the asperities, are measured and calculated from one cross-section profile from the $10 \times 10 \mathrm{\mu m}^{2} \mathrm{AFM}$ scan data. The accuracy of these parameters can be increased by taking all 3D AFM scan data into account.

An IR camera was used to monitor the initial bonding process. The bond wave propagation speed was measured. Immediately after room temperature bonding, the bond energies were measured using the crack-opening method [15].

The bonded wafer pairs were debonded. After another cleaning cycle, the wafers were bonded again. The bond wave propagation speed and the bond energies were measured once more.

Finally, the bonded wafers were debonded once more. The wafers were cleaned with fuming nitric acid $(100 \%)$ and hot nitric acid $\left(70 \%\right.$ at $\left.90^{\circ} \mathrm{C}\right)$, followed by a QDR with DI water. Samples were prepared from these wafers for surface roughness examinations. An
AFM was used for measuring the wafer surface roughness.

As shown in Table 1, wafers Nos. 6 and 7 have been polished using two different CMP processes. The values of the standard deviation of the asperity height of these two wafers are about 10 times higher than that of the original wafer surface (similar to the surface of wafer No. 10). The mean radii of the asperity caps become about 7 times smaller. However, there are fewer asperities per unit area on these two wafers. In general, the surface of wafer No. 6 is slightly rougher than that of wafer No. 7 .

After shortly etching in an HF solution and a $\mathrm{KOH}$ solution, the surfaces of wafers Nos. 8 and 9 become significantly rougher than before. Though the values of the standard deviation are more or less the same as those of wafers after CMP, the mean cap radii are much smaller than those of wafers Nos. 6 and 7. The density of the asperities is much higher.

It is worthwhile mentioning that the values, $R \sigma \eta_{s}$, for each wafer are almost equal to a constant, close to 0.07 . This result also implies that the value $R, \sigma$, and $\eta_{s}$, have been correctly extracted.

Table 2 Results of direct wafer bonding at room temperature

\begin{tabular}{||l||l|l|l|l||}
\hline $\begin{array}{l}\text { Bonded } \\
\text { wafer } \\
\text { pairs }\end{array}$ & $\theta$ & Bond speed & $\begin{array}{l}\text { bonding } \\
\text { energy } \\
{\left[\mathrm{J} / \mathrm{m}^{2}\right]}\end{array}$ & Voids \\
\hline \hline 1 and 6 & 9.5 & $\begin{array}{l}\text { With pressure, } \\
\text { slow }\end{array}$ & 0.05 & a few \\
\hline 2 and 7 & 7.7 & slow & 0.07 & a few \\
\hline 3 and 8 & 16.8 & Not bondable & - & - \\
\hline 4 and 9 & 13.8 & Not bondable & - & - \\
\hline 5 and 10 & 0.1 & Spontaneously & 0.10 & No \\
\hline
\end{tabular}

Note: I When calculating $\theta$, Young's modulus of single crystalline silicon $\langle 100\rangle$ is $130 \mathrm{GPa}$, the Poisson ratio is 0.28 , and the specific surface energy of a hydrophilic silicon wafer surface is $0.1 \mathrm{~J} / \mathrm{m}^{2}$.

2 Wafer Nos. 1 to 5 are from the same batch as No. 10. They are assumed to have the same surface roughness as that of wafer No. 10.

The results of direct wafer bonding at room temperature are summarised in Table 2 . Correlation between the adhesion parameter and the effective bonding energy or the bond speed is clear. Wafer pair $5+10$, which has the lowest adhesion parameter $(0.1$, below the critical value), bonded spontaneously, yielding the highest specific effective bonding energy of $0.10 \mathrm{~J} / \mathrm{m}^{2}$, that is the same as the surface energy of adhesion for hydrophilic silicon surfaces. Wafers pairs $3+8$ and $4+9$ were not bondable at all, even under pressure. Wafer pair $1+6$ bonded very slowly and with the use of pressure. Wafer pair $2+7$ bonded slowly after being initiated with the application of slight pressure. The effective bonding energies for wafers pairs $1+6$ and $2+7$ are low. 
For wafer pairs $1+6$ and $2+7$, there are a few voids that are visible by means of the IR camera. However, these can not be seen as the measurements of the real area of contact, because there may be microvoids that are not detectable using the IR inspection method.

A discrepancy between the experimental results and the model can also be observed. According to Fig. 8, the specific effective bonding energy for wafer pair $1+6$ and $2+7$ should be not as high as 0.05 or $0.07 \mathrm{~J} / \mathrm{m}^{2}$, or the values of the surface adhesion parameter for these two wafer pairs should be not as high as 9.5 or 7.7. This discrepancy may be caused in the bonding energy measurements, in the surface roughness experiments, or in the calculation of the surface adhesion parameters.

\section{CONCLUSION}

Based on the DMT - Maugis theory on the contact and adhesion between elastic bodies, the real area of contact after room temperature direct wafer bonding is calculated. The real area of contact in the wafer bonding interface depends solely on one dimensionless parameter, viz. the surface adhesion parameter. In this parameter, the specific energy of adhesion, the material elasticity, and the surface roughness characteristics, such as standard deviation of the asperity height, the mean cap radius of the asperity, are included. It has been theoretically proved that a full area of contact is achievable during room temperature wafer bonding provided that the wafer surface adhesion parameter is smaller than a critical value.

The mechanical energy used in the separation per unit area of bonded wafer interface is defined as the specific effective bonding energy, the maximum value of which is the specific energy of adhesion. The influence of surface roughness in the specific effective bonding energy has been studied. The specific effective bonding energy also depends solely on the wafer surface adhesion parameter.

Using the surface adhesion parameter as a measure, three kinds of wafer contact interfaces can be identified with respect to its bondability: the non-bonding regime $(\theta>12)$, the bonding regime $(\theta<1)$, and the adherence regime $(l<\theta<12)$. If the wafers are in the nonbonding regime, the real area of contact and the effective bonding energy will be approximately zero, but should they be in the bonding regime, full area of contact and the specific energy of adhesion are achievable.

Using CMP and chemical etching, wafer surfaces, have been modified such that they have a slight roughness difference on a microscopic scale. Direct bonding experiments on such wafers have been conducted. The correlation between the surface adhesion parameter and the measured effective bonding energy or the bond speed are in reasonable agreement with theory.

The relation between bonding, stiction and friction is quite close. The theory presented here will have consequences for surface micromachining and the dynamics of the micromechanical systems with a sliding contact.

Acknowledgement This research has been financially supported by the Dutch Technology Foundation (STW)

\section{REFERENCES}

[1] Q. -Y. Tong, and U. Gösele, Semiconductor wafer bonding: recent developments, Materials Chemistry and Physics, 37, 101 - 127, (1994).

[2] R. Stengl, K. Mitani V. Lehmann, and U. Gösele, Silicon wafer bonding: chemistry, elasto-mechanics, and manufacturing, Proc. IEEE SOS/SOI Tech. Conf. Pp. 123 124 (1989).

[3] U. Gösele, and Q. -Y. Tong, Silicon layer transfer by wafer bonding, Proc. $2^{\text {nd }}$. Int. Symp. Semiconductor Wafer Bonding: Science, technology, and Applications, M. A. Schmidt, T. Abe, C. E. Hunt, and H. Baumgart, Editors, PV 93-29, The Electrochemical Society Proceedings Series, Pennington, NJ, pp. 395 - 409 (1993).

[4] Q. -Y. Tong, and U. Gösele, Thickness considerations in direct wafer bonding, J. Electrochem. Soc., Vol. 142, No. 11, pp. 3975 - 3979 (1995).

[5] $\mathrm{H}$. Yu and $\mathrm{Z}$. Hu, A model of wafer bonding by elastic accommodation, J. Mech. Phys. Solids, Vol. 46, No. 5, pp. 829 - 844 (1998)

[6] J.F. Archard, Surface topography and tribology, Tribology Int., 7, 213 - 220 (1974).

[7] B. V. Deryagin, V. M. Muller, and Yu. P. Toporov, Effect of contact deformation on the adhesion of particles, J. Colloid Interface Sci. Vol. 53, No. 2, pp. 314 - 326 (1975).

[8] D. Maugis, Adhesion of spheres: the JKR - DMT transition using a Dugdale model, J. of Colloid and Interface Sci., Vol. 150, No. 1, 243 - 269 (1991).

[9] K. L. Johnson, A continuum mechanics model of the adhesion and friction in a single asperity contact, to be published.

[10] D. Maugis, On the contact and adhesion of rough surfaces, J. Adhesion Sci. Technol., Vol. 10, No. 2, pp. 161 $175(1996)$

[11] K. N. G. Fuller, and D. Tabor, The effect of surface roughness on the adhesion of elastic solids, Proc. R. Soc. Lond. A. 345, 327 - 342 (1975).

[12] Q. - Y. Tong, T. H. Lee, U Gösele, M. Reiche, J. Ramm and $E$, Beck, The role of surface chemistry in bonding of standard silicon wafers, J. Electrochem. Soc., Vol. 144, No. 1, pp. 384 - 389 (1997).

[13] R. Stengl, T. Tan, and U. Gösele, A model for the silicon wafer bonding process, Jap. J. Appl. Phy., Vol. 28, No. 10, pp. $1735-1741$ (1989).

[14] C. Gui, H. Albers, J. G. E. Gardeniers, M. Elwenspoek, P. V. Lambeck, Fusion bonding of rough surface with polishing technique for silicon micromachining, $\mathrm{J}$. Microsystem Technologies, Vol. 3, No. 3, 122 - 128 (1997).

[15] W.P. Maszara, G. Goetz, A. Cavilia, and J.B. McKitterichk, Bonding of silicon wafers for silicon-oninsulator, J. Appl. Phys., 64, 4943 (1988). 